\title{
Total Sleep Deprivation and Weight Increases of the Reproductive Organs in Male Rats
}

\author{
SHoJiro INOUE, HiroAKI NAGASAKI AND MASAMI IRIKI \\ Institute for Medical and Dental Engineering, \\ Tokyo Medical and Dental University, Tokyo 101, \\ and Department of Physiology, Tokyo Metropolitan \\ Institute of Gerontology, Tokyo 173
}

\begin{abstract}
Synopsis
Long-term total sleep deprivation was applied to male rats using a special cage, in which the rats were continuously forced to move in order to avoid an electric shock through the floor. In comparison with untreated control, three groups of 24-, 48- and 72-hr sleep-deprived rats showed marked weight increases in the adrenals (30.8-47.7\%), testes $(6.2-15.0 \%)$, ventral prostates $(59.7-90.7 \%)$ and seminal vesicles $(34.2-40.1 \%)$. It is supposed that either the sleep deprivation per se or stressful conditions accompanied with the treatment caused a hypersecretion of hypophyseal and testicular hormones.
\end{abstract}

During normal sleep, some hormones especially from the hypophysis and the adrenal cortex are known to show special patterns of fluctuations in the blood level (see Weitzman, 1972). However, no systematic investigation has ever been reported as to the relationship between sleep loss and endocrine activities. Meanwhile, during the course of physiological and biochemical studies on sleep mechanisms, which will be published elsewhere (Nagasaki et al., 1974), the present authors noticed that 24-hr sleep-deprived rats exhibited a marked increase in the size of the reproductive organs. On the basis of this finding, an attempt was carried out to analyze quantitatively the effect of long-term total sleep deprivation on several organ weights. Here the results are dealt with.

\section{Materials and Methods}

Twenty-four male rats of the Sprague-Dawley strain, 6-7 months of age, kept at a controlled environment $\left(24^{\circ} \mathrm{C} ; 50 \%\right.$ humidity; lights on $8: 00$

Received for publication February 19, 1974.
20:00) since birth, were used. Six rats were killed by a blow on the neck without treatment (normal control). The others were placed together in a special cage for total sleep deprivation. The cage is composed of 4 rooms connected by gates. Any one floor out of the 4 rooms is automatically passed by an electric current of $60 \mathrm{sec}$ duration at regular intervals of $180 \mathrm{sec}$, while the other three are freed from the stimulus. The electric shock is applied sequentially to every room. Each room is illuminated by a ceiling light during the current passage. This light turns on and off $5 \mathrm{sec}$ in advance to indicate that the shock is coming and leaving, respectively. Thus rats can easily learn how to avoid the shock, moving from one room to another without sleeping. The details of the cage are described by Ichikawa et al. (1973). Rat chow and water were given ad libitum. Six rats in each group were killed after 24-, 48- and 72-hr sleep deprivation. Autopsies were performed between 9:30 and 10:30. Body weights were recorded before and after the treatment. Weights of the hypophysis, adrenals, ventral prostates, seminal vesicles and testes were weighed by an analytical balance with the accuracy up to $0.1 \mathrm{mg}$.

\section{Results}

In 24-hr total sleep deprivation, all rats lost their body weight by $14.5-27.8 \mathrm{~g}$ (in average $20.8 \mathrm{~g}$ ), which corresponds to $2.31-5.23 \%$ 


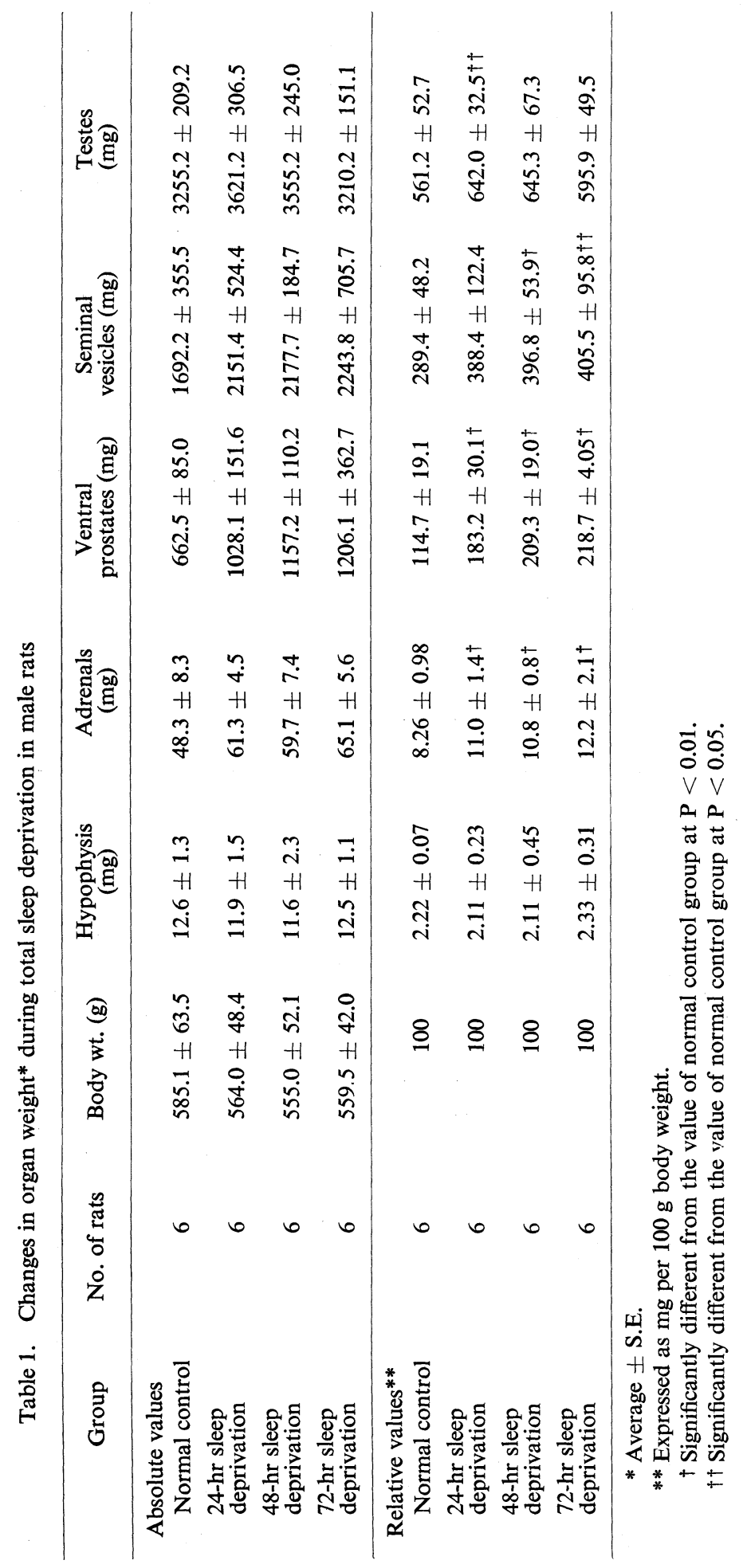


(in average $3.59 \%$ ) of the initial weight. Three 48-hr sleep-deprived rats lost their body weight by $3.6-16.9 \mathrm{~g}$, while the other three gained by $0.4-14.6 \mathrm{~g}$. The average of this group is a decrease of $2.6 \mathrm{~g}(0.60 \%)$. In $72-\mathrm{hr}$ sleep deprivation group too, a half of the members showed a body weight gain of $2.2-$ $12.9 \mathrm{~g}$, while the rest half showed a loss of $6.7-50.7 \mathrm{~g}$. Two rats of the latter members died shortly before the termination, presumably due to exhaustion. The average of this group is a loss of $8.2 \mathrm{~g}(1.50 \%)$. Consequently, the body weight loss caused by the initial 24-hr sleep deprivation almost recovered in the following $24 \mathrm{hr}$. Sleep-deprived rats exhibited from the 2 nd day on a vigorous appetite, eating and drinking even in the daytime. They became considerably furious on the 3rd and 4th days, otherwise very tame.

Organ weights are summarized in Table 1. Statistical analyses were applied to the relative values. The hypophyseal weights of all sleepdeprived groups were not significantly different from that of the control group. However, the weights of the adrenals and the ventral prostates were greatly heavier in all treated groups, showing an increment of $30.8-47.7 \%$ and $59.7-90.7 \%$, respectively, as compared to the values of the normal group. The seminal vesicles were also heavier in all sleep deprivation groups (an increment of $34.2-40.1 \%$ ). However, the value of the $24-\mathrm{hr}$ treated group was not statistically significant because of large individual variations. The testes became also heavier in all treated groups (an increment of $6.2-15.0 \%$ ), but only the 24-hr sleep deprivation group showed a statistical difference from the normal group.

\section{Discussion}

It was found that long-term total sleep deprivation induced marked increases in the weights of the adrenals, ventral prostates, seminal vesicles and testes of adult rats.

The enlargement of the adrenals may be due to stressful conditions such as the sleep deprivation by itself, continuously forced locomotion, electric shock through the floor and/or a photic stimulation by the on-off repetitions of the ceiling indicator lights. The furious behavior and the exhaustion death of sleepdeprived rats observed on the 4th day of the treatment may be accounted for by the adrenocortical hyperfunction.

Weight increases in the accessory reproductive organs of sleep-deprived rats might be caused by an androgenic stimulation. Factors involved in the sleep deprivation treatment seem to have activated the brain-hypophyseal system to bring about an increase in the secretion of not only ACTH but also gonadotropins and prolactin, which stimulated androgen release from the testes. It may be possible to assume that the sleep deprivation by itself directly caused the activation of the brain-hypophyseal-testicular function. Another possible explanation is that the continuous photic stimulation by the indicator lights might trigger the increased gonadotropin secretion, since constant illumination is known to induce sometimes an elevation of hypophyseal gonadotropic activities. Dunn et al. (1972) reported that in the male rat a stressful condition can induce an increased $\mathrm{LH}$ and prolactin release from the hypophysis. In this connection, there is a possibility that all the above-mentioned stressful conditions were responsible also for the weight increases of the reproductive system. Further analyses of the mechanism are now in progress.

\section{Acknowledgement}

The authors would like to express their cordial thanks to Professor Koji Uchizono, Department of Physiology, Faculty of Medicine, University of Tokyo, for his invaluable discussion and encouragement.

\section{References}

Dunn, J. D., A. Arimura and L. E. Scheving (1972). Endocrinology 90, 29. 
Ichikawa, H., K. Honda and S. Inoué (1973) Reports Inst. Med. Dent. Eng. (Tokyo Med. Dent. Univ.) 7, 145. (In Japanese)

Nagasaki, H., M. Iriki, S. Inoué and K. Uchizono (1974). Proc. Japan Acad. 50, 241.
Weitzman, E. D. In The Sleeping Brain, Perspectives in the Brain Sciences Vol. 1, ed. M. H. Chase, Brain Information Service/Brain Research Institute, UCLA, Los Angeles, 1972, p. 193. 